\title{
A heart-adipose tissue connection in the regulation of energy metabolism
}

\author{
Sheila Collins
}

\begin{abstract}
Almost 20 years ago, the protein encoded by the ob locus in mice was identified as an adipocytesecreted hormone, now termed leptin, which functions as a peripheral signal to communicate the organism's energy reserve-and thereby protects against starvation due to insufficient caloric resources. Additional peripheral factors have since been identified that coordinate interorgan crosstalk to manage energy resources. The heart is included in this network through its regulated release of natriuretic peptides A and B-cardiac hormones originally identified as important in blood pressure control. Emerging evidence that natriuretic peptide receptors are expressed in adipose tissue, and that circulating levels of these peptides are decreased in animals and humans with obesity, could imply that natriuretic peptides are also involved in the regulation of energy metabolism. The natriuretic peptides stimulate triglyceride lipolysis in adipocytes, a process also regulated by the sympathetic nervous system. In addition, these two pathways promote uncoupling of mitochondrial respiration and thermogenesis in brown adipocytes. This Review focuses on the roles of the natriuretic peptides and the sympathetic nervous system in regulating adipocyte metabolism. The potential for manipulating the natriuretic peptide pathway to increase energy expenditure in obesity and manage the complications of cardiometabolic disease is also discussed.
\end{abstract}

Collins, S. Nat. Rev. Endocrinol. advance online publication 3 December 2013; doi:10.1038/nrendo.2013.234

\section{Introduction}

The heart and adipose tissue are linked through the now well-appreciated fact that obesity is a risk factor for heart disease, including coronary artery disease and hypertension. ${ }^{1,2}$ Additional common themes between these two organs include the fact that key functions of adipose tissue (lipolysis) and the heart (rate and force of contraction) are regulated by the sympathetic nervous system (SNS) through $\beta$-adrenergic receptor signalling. Consequently, disease-related perturbations in content and activity of the $\beta$-adrenergic receptors can impair these functions. Activation of lipolysis in adipose tissue, in turn, provides fatty acids to the heart as metabolic fuel. Moreover, beyond these simple parallels, evidence has been steadily growing that the heart can directly regulate adipose tissue through cardiac natriuretic peptides A and B: ANP and BNP, respectively. This Review discusses current views of how adipose tissue metabolism is regulated by natriuretic peptides, including the functions of white and brown adipocytes. Unanswered questions are also highlighted, including those relating to the full range of tissues in which natriuretic peptides might control energy balance.

Diabetes and Obesity Research Center, Sanford-Burnham Medical Research Institute, 6400 Sanger Road, Orlando, FL 32827, USA. scollins@ sanfordburnham.org

\section{Regulation of fat metabolism \\ Receptors for ANP and BNP in adipose tissue}

ANP and BNP are best known for their action in the kidney to promote diuresis and natriuresis, and they are thought to also act through autocrine mechanisms to

Competing interests

The author declares no competing interests. protect the heart from high preload and afterload pressures, which can result in hypertrophy and fibrosis. However, emerging evidence suggests that ANP and BNP have an important additional role in the regulation of metabolism and energy expenditure. ${ }^{3}$ Almost two decades ago, researchers interested in the relationship between obesity and hypertension noted that the receptors for ANP and BNP were expressed in human adipose tissue (Figure 1), ${ }^{4}$ and that obesity in patients seemed to be associated with decreased circulating levels of natriuretic peptides and with altered levels of expression of the corresponding receptors. ${ }^{5,6}$ In particular, levels of atrial natriuretic peptide receptor 3 (NPR-C), the so-called clearance receptor, are markedly elevated in both humans and rodents with obesity. 7.8 These observations fuelled speculation that adipose tissue was a site of natriuretic peptide clearance. Although as yet no evidence exists to support or refute this notion, increased clearance of natriuretic peptides in adipose tissue could potentially contribute to both reduced circulating levels of natriuretic peptides and predispose to hypertension in individuals with obesity. Interestingly, obesity per se, irrespective of blood pressure, was associated with reductions in plasma levels of natriuretic peptides, including $\mathrm{N}$-terminal pro-ANP, ${ }^{6}$ indicating that synthesis and processing of natriuretic peptides is also defective in individuals with obesity.

Roles of ANP and BNP in adipocyte metabolism More than a decade ago, ANP was demonstrated to directly affect adipose tissue by stimulating lipolysis in 


Key points
- The heart is included in the network of endocrine organs that regulate energy
usage and metabolism
- The cardiac natriuretic peptide system and the sympathetic nervous system
should be considered dual regulators of energy metabolism
- Improved understanding of energy expenditure regulation by neurohormonal
signals in adipose tissue might aid in finding ways to manipulate these
processes
- Improved understanding of receptors for the natriuretic peptides at the
molecular level is needed, because these receptors are dynamically regulated
and dictate responses to these peptides
- Environmental factors (ambient temperature, diet and physical activity)
coordinate metabolic fuel management in adipose tissue and muscle through
both sympathetic nervous system tone and cardiac sensing of blood pressure

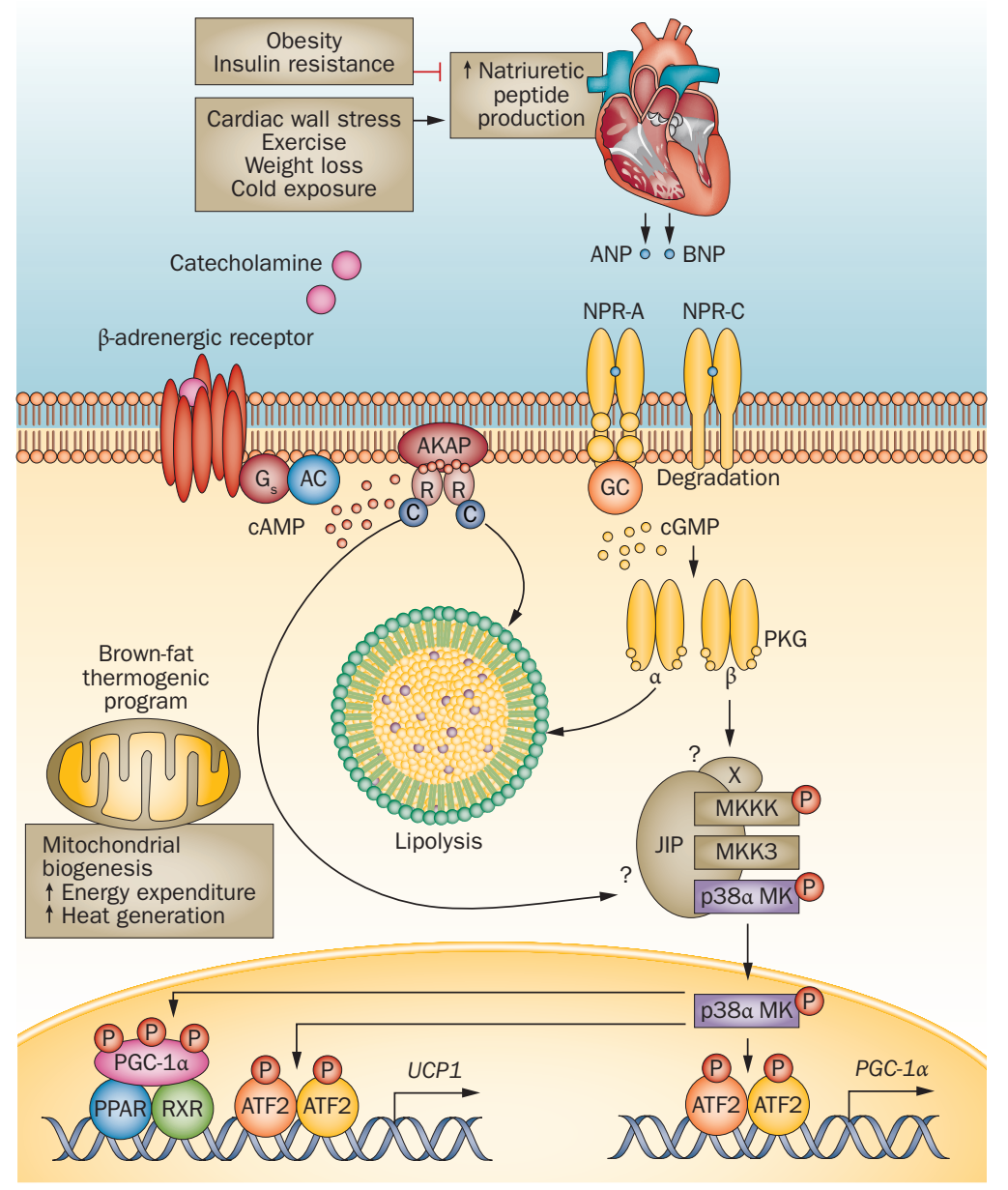

Figure 1 | Cardiac natriuretic peptide signalling in adipocytes. The natriuretic peptides ANP and BNP are produced by the heart and regulate adipocyte lipolysis by binding to two homodimeric receptors, NPR-A and NPR-C. Binding of natriuretic peptides to NPR-A activates guanylyl cyclase, producing cGMP, which activates cGMP-dependent protein kinase (PKG). In turn, PKG-mediated phosphorylation triggers a signalling cascade that results in enhanced lipolysis and activation of p38 mitogen-activated protein kinase, as well as inducing the brown-fat thermogenesis pathway. In parallel with this mechanism, $\beta$-adrenergic signalling activates adenylate cyclase, producing CAMP. Binding of CAMP to the regulatory subunits of cAMP-dependent protein kinase releases its catalytic subunits, initiating a signalling cascade that also independently stimulates these two pathways. The socalled clearance receptor, NPR-C, binds and internalizes natriuretic peptides, which targets them to be degraded. The ratio of NPR-A to NPR-C is, therefore, an important determinant of the strength of NPR-A-PKG signalling. cultured human adipocytes, through a parallel pathway to catecholamine-mediated lipolysis (Figure 1). ${ }^{9}$ Further clinical studies showed that levels of circulating free fatty acids increase in response to ANP, resulting in an increase in energy expenditure attributable to postprandial lipid oxidation. ${ }^{10-12}$ The mobilized free fatty acids released from adipose tissue could be consumed in the liver, ${ }^{12}$ although adipose tissue itself and skeletal muscle are equally likely to be sites of fatty acid oxidation. ${ }^{13-15}$

\section{Energy expenditure in adipose tissue}

Studies of energy expenditure in humans, and approaches to increase it, have predominantly focused on increasing fuel oxidation in skeletal muscle through inducing cellular adaptations to exercise, ${ }^{16}$ particularly given that skeletal muscle represents a large proportion of total body mass. This approach remains very viable; for example, the possibility that uncoupling of mitochondrial respiration in skeletal muscle might be exploited to increase energy expenditure is being explored. ${ }^{17}$ Similarly, established mechanisms of increasing energy expenditure exist in adipocytes, including adaptive thermogenesis in brown adipocytes. Furthermore, white adipocytes are now appreciated to be capable of substantial oxidative respiration, a portion of which can be uncoupled. ${ }^{15}$

\section{Brown adipocytes}

Brown adipose tissue (BAT) was initially thought to be a specialized gland involved in hibernation, although by the beginning of the $20^{\text {th }}$ century researchers had realised that it was a type of adipose tissue. ${ }^{18}$ BAT came to be appreciated as an important primeval organ designed to generate heat using metabolic fuel, ${ }^{19}$ which had enabled our ancestors to survive low winter temperatures long before houses and clothes existed. In the mid- $20^{\text {th }}$ century the ability of BAT to burn calories in heat generation was proposed as a way to reduce excess body fat, leading to excitement over using the energy-expending potential of BAT to combat obesity and associated metabolic disorders. ${ }^{20}$ However, by the early 1980 s the notion that BAT had any appreciable role in adult humans was dismissed. The prevailing view was that although newborn humans possess discernible BAT, it rapidly disappears with age and is irrelevant to adult human physiology-despite the fact that human autopsy studies showed that small amounts of BAT persisted into the $6^{\text {th }}$ decade of life or more. ${ }^{21}$ In essence, BAT was relegated to the status of a vestigial organ in humans. ${ }^{22}$

In the past few years, the (re)discovery that metabolically active BAT exists in adult humans ${ }^{23-28}$ has now been reinforced by observations that BAT activity is inversely correlated with BMI, fat mass and insulin sensitivity. ${ }^{29,30}$ Given the paucity of successful and safe pharmacological therapies for obesity, including the general failure of extensive research efforts targeting the control of appetite and feeding behaviour in the central nervous system, the mechanisms that control peripheral energy metabolism (including activation of BAT) are again receiving attention for their potential to raise net energy expenditure. ${ }^{31,32}$ 
Box 1 | The missing key

Two studies in humans ${ }^{80,81}$ showed that, unlike cold exposure, administration of sympathomimetics such as ephedrine or isoprenaline does not increase the activation of brown fat (as shown by uptake of ${ }^{18} \mathrm{~F}$-fluorodeoxyglucose on PET imaging). This interesting and somewhat unexpected finding could be accounted for by several possibilities: that local concentrations of systemically infused agents did not reach the thresholds needed to activate brown fat cells (although they did elicit other expected parameters of elevated sympathetic nervous system activity); or that additional factors triggered by the cold stimulus are needed to cooperatively activate brown (or beige) adipocytes in conjunction with $\beta$-adrenergic stimulation.

For example, $\beta$-adrenergic agonists and natriuretic peptides might have additive or even synergistic effects on the expression of UCP1 or PGC-1a. Moreover, depending upon the neuronal location, various neuropeptides can be co-secreted with neurotransmitters. In the case of catecholamines, these can include neuropeptide $\mathrm{Y}$, somatostatin and calcitonin gene-related peptide, among others. Such an arrangement might only induce signalling when two stimuli are present together-like a safe deposit box that requires two keys to allow it to be unlocked. More studies are needed to confirm the existence of this disparity between the effects of cold exposure and sympathomimetic agents. However, if it is real, it would seem that finding the missing key could lead to genuine treasure.

\section{White adipocytes}

White adipose tissue (WAT) is primarily an energy storage depot and the source of free fatty acids and glycerol released by lipolysis into the circulation, to provide fuel for utilization by other tissues in the body. As such, WAT has not traditionally been viewed as a site of energy expenditure. However, in addition to being released into the circulation (and, to a much lesser extent, being re-esterified in $s i u^{33}$ ), a portion of the liberated free fatty acids undergo mitochondrial oxidation within WAT itself. ${ }^{15,34,35}$ In addition, emerging evidence indicates that WAT has a higher mitochondrial content than was previously believed. ${ }^{34,36}$ Together, these findings highlight a potentially important role for white adipocytes in the regulation of whole-body physiology. ${ }^{37}$ Although basal levels of mitochondrial fatty acid oxidation are much lower in WAT than in either BAT or skeletal muscle, this mechanism should not be overlooked, as it could represent a considerable contribution to energy expenditure, especially in individuals with obesity, owing to their large total mass of adipose tissue. ${ }^{34,38}$ Understanding of the mechanisms underlying this UCP1-independent process, and how to harness this aspect of energy expenditure, should be further pursued. The contribution of natriuretic peptides (and perhaps other factors) to fatty-acid-dependent oxygen consumption resulting from lipolysis in WAT will, therefore, be important to explore.

\section{Activation of BAT and browning of WAT $\beta$-adrenergic signalling}

The SNS is well-established as an activator of adipocyte lipolysis (mediated by the release of catecholamines) as well as uncoupled respiration for nonshivering thermogenesis in brown adipocytes. ${ }^{39}$ Sympathetic activation is also an important stimulus for the development of adipocytes with a brown phenotype (also known as brite or beige adipocytes) within WAT depots. ${ }^{40}$ The source and identity of these brite or beige adipocytes is the subject of intensive research in several laboratories. ${ }^{41-43}$ Of note, it is possible that multiple sources of beige or brite adipocytes exist that depend on the location of the fat depot and the nature of the stimulus.

\section{Natriuretic peptide signalling}

My research group found that natriuretic peptides not only stimulate lipolysis but also activate brown adipocytes, as well as participating in the 'browning' of adipocytes in WAT. ${ }^{13}$ Moreover, natriuretic peptides, by activating atrial natriuretic peptide receptor 1 (NPR-A), can cooperate with catecholamines acting via $\beta$-adrenergic receptors to stimulate parallel pathways involving cGMP-dependent protein kinases (PKG) and CAMP-dependent protein kinase (PKA), respectively. ${ }^{13}$ In addition to promoting lipolysis through PKA and PKG, these two signals converge on p38 mitogen-activated protein kinase to orchestrate a transcriptional response resulting in increased mitochondrial biogenesis, elevated expression of UCP1 and PGC-1 $\alpha$, and probably also other steps that ramp up uncoupled respiration and thermogenesis. ${ }^{13,44}$ In addition, the ability of natriuretic peptides to stimulate lipolysis also supports brown adipocyte thermogenesis by releasing fatty acids, which activate UCP1 and provide fuel for mitochondrial respiration in brown adipocytes. ${ }^{9}$ Although in vivo the SNS and the natriuretic peptide systems probably work together in an integrated manner under physiological conditions, the metabolic effects of natriuretic peptides persist during systemic $\beta$-adrenergic blockade, which supports the argument that natriuretic peptides have a direct and specific role in energy metabolism. ${ }^{11}$

Cold exposure has been used for many years as the classic stimulus to induce sympathetic activation, thereby promoting nonshivering thermogenesis and increased energy expenditure in BAT (Box 1). So my research group was initially surprised to see that animals exposed to cold exhibited increased expression of ANP and BNP in the heart, increased circulating levels of BNP, and a shift in the expression of the two natriuretic peptide receptors in adipose tissue favouring activation of the natriuretic peptide system, in conjunction with the expected activation of the SNS. ${ }^{13}$ However, cold exposure in rodents and humans can significantly increase blood pressure, ${ }^{45}$ ANP expression in the heart, and plasma levels of ANP. ${ }^{46}$ Although some evidence suggests that the changes in natriuretic peptide levels are direct responses to SNS output to the heart, ${ }^{47}$ the effect on blood pressure probably results from a rise in central blood volume in response to skin vasoconstriction, with the increase in cardiac filling pressure triggering the secretion of ANP.

The molecular basis for the changes in adipose tissue expression of the receptors NPR-A and NPR-C in response to cold exposure is not clear but might be influenced by sympathetic tone, given that a similar shift in the NPR-A to NPR-C ratio in adipose tissue is also observed in response to selective activation of $\beta_{3}$-adrenergic receptors, expression of which is confined to adipose tissue in mice. ${ }^{48,49}$ By contrast, in the context of the increased expression of NPR-C that occurs in individuals with obesity, an interesting observation (in both rodents and humans) is that elevated insulin levels have been linked 
to increased expression of NPR-C in adipose tissue..$^{50,51}$ Clearly, with this limited understanding of how the natriuretic peptide receptors are regulated in adipose tissue, further research is required, including studies of the transcriptional regulation of NPRA and NPRC genes, the effects of post-translational modifications that can alter the function of these receptors, such as phosphorylation, and the dynamics of receptor endocytosis and recycling. ${ }^{52,53}$ Notably, the amount of NPR-C in adipose tissue is much higher in rodents than in humans. ${ }^{54}$ Nevertheless, despite this difference in absolute levels of NPR-C expression between rodents and humans, the patterns of changes in its expression induced by diet, fasting and other stimuli are similar in these two species. ${ }^{54}$

\section{Metabolic effects outside adipose tissue}

The role of natriuretic peptides in energy metabolism might not be confined to adipose tissue. Cultured human skeletal muscle myotubes have been reported to respond to exogenous ANP and BNP with modest but significant increases in the expression of the genes encoding PGC-1a and components of the oxidative phosphorylation cascade, as well as with increased fatty acid oxidation. ${ }^{14}$ In a preliminary study, my research group observed that quadriceps muscle from $\mathrm{Nprc}^{-/-}$mice had increased palmitate oxidation (S. Collins et al., unpublished work), but this finding will need to be investigated further. As is the case for most other tissues, the regulation of NPRA and NPRC expression in skeletal muscle is not well understood, but in one study modest increases in NPR-A transcripts were observed in response to exercise training. ${ }^{14}$ This observation is interesting, because the coordinated increases in SNS activity, heart rate, blood pressure and release of natriuretic peptides observed in some forms of exercise might communicate the need to access stored nutrients, such as fatty acids, from adipose tissue for consumption by the heart and skeletal muscle.

Other tissues that might be metabolically affected by natriuretic peptide signalling include the vasculature, through its close juxtaposition to perivascular adipose tissue, which displays characteristics of BAT, including the expression of natriuretic peptide receptors. ${ }^{55-57}$ A growing literature describes how the metabolic activities of perivascular adipose tissue are affected by diabetes mellitus, endothelial dysfunction and cardiovascular disease. ${ }^{57-59}$ Further studies are required to shed light on perivascular adipose tissue biology, in particular whether the natriuretic peptide receptors in perivascular adipose tissue are regulated in response to nutritional and environmental status, and how circulating natriuretic peptides (and other factors) might affect the function of perivascular adipose tissue and that of the closely apposed endothelia.

\section{Cardiac cachexia}

Most of this Review has focused on the potential positive aspects of how cardiac natriuretic peptides might promote increased energy expenditure in adipose tissue, including through the browning of WAT. However, a possible adverse effect has been noted concerning the development of cachexia in patients with heart failure. A small fraction of patients with heart failure develop cardiac cachexia, which is generally defined as unintentional, non-oedematous weight loss of $>6 \%$ over a 6 -month period, and is a serious complication with a fairly poor prognosis. ${ }^{60}$ The weight loss has been typically reported to consist of both fat and lean mass. ${ }^{61}$ Many factors are implicated in the mechanisms underlying cardiac cachexia, including sympathetic tone and increased levels of proinflammatory cytokines. ${ }^{62}$ However, evidence from prospective studies indicates that the decrease in fat mass precedes that of lean mass. ${ }^{63-65}$ Moreover, individuals with cardiac cachexia have exceedingly high circulating levels of BNP, including a significant fraction of incompletely processed $\mathrm{N}$-terminal pro-BNP.

The fact that natriuretic peptides can increase lipolysis and energy expenditure in adipose tissue, as well as inducing some browning of WAT, raises the question of whether the elevated natriuretic peptide levels in patients with heart failure might contribute to excessive weight loss in patients who develop cachexia. ${ }^{12,66}$ However, experiments to test this hypothesis could prove challenging to perform. For example, from a practical standpoint, fragile cachectic patients with decompensated congestive heart failure might not be able to lie flat for long enough to participate in ${ }^{18} \mathrm{~F}$-fluorodeoxyglucose PET imaging studies, which are currently the best approach for measuring the activation of BAT (by identifying foci of significantly increased tracer uptake in areas where BAT is present). In addition, patients with cardiac cachexia often exhibit heightened sympathetic tone, ${ }^{62}$ which would increase ${ }^{18} \mathrm{~F}$-fluorodeoxyglucose uptake, confounding the interpretation of PET imaging results. Obtaining indirect calorimetry measurements (to detect changes in the basal metabolic rate under isocaloric conditions) in a sufficient number of such patients might be feasible. However, without the PET imaging studies it would not be possible to determine the location of any increases in energy expenditure. As such, proposing to put these very ill patients through such experimental procedures presents an ethical dilemma.

If cardiac cachexia does involve heightened energy expenditure due to elevated natriuretic peptide signalling (resulting in browning of WAT), and if fatty acid oxidation is thereby increased in the adipose tissue and skeletal muscle of these patients, the therapeutic challenge will be to block these metabolic effects of the excess natriuretic peptides without obliterating the natriuretic and diuretic benefits these hormones provide.

\section{Future perspectives}

Potential clinical benefits might be gained from the ability of natriuretic peptides to increase lipolysis, browning of WAT (as well as the uncoupled respiration that accompanies it), and possibly also skeletal muscle fuel oxidation (Figure 1). Research is at too preliminary a stage to know for sure, but data obtained in humans offer some cautious optimism. For example, the presence of detectable amounts of active brown adipocytes in humans has been linked to increased energy expenditure, improved glycaemic control and reduced fat mass. ${ }^{19,29,67,68}$ Moreover, 
pharmacologically improving insulin sensitivity significantly improves the ratio of NPRA:NPRC in adipose tissue, but not in skeletal muscle (S. Collins et al., unpublished work). Similarly, increased circulating levels of natriuretic peptides in humans have been correlated with decreased body fat content, decreased risk of type 2 diabetes mellitus, and profiles of circulating factors associated with improved insulin sensitivity. ${ }^{69-71}$

To take this work forward, direct interventional studies in humans are now needed to assess the metabolic effects of natriuretic peptide infusions, some of which could be given chronically over several days to weeks. Previous studies of the effects of infusions of natriuretic peptides in both animals and humans have largely focused on blood pressure, cardiac responses and kidney function (although some studies ${ }^{10-12}$ have assessed the effects of natriuretic peptide infusion on energy utilization, in terms of the balance between fat and carbohydrate oxidation). However, similar studies looking at metabolism as well as the mechanisms that might be controlling these metabolic responses are not yet available. Such studies should include assessments of changes in adipose tissue and muscle metabolism, and evaluation of the gene expression profiles of these two tissues with regard to the expression of natriuretic peptide receptors, mitochondrial respiration proteins and brown adipocyte markers. These investigations will have to be done carefully, considering the potent effects of natriuretic peptides on natriuesis and diuresis. Modulation of natriuretic peptide levels could also produce feedback changes in components of the renin-aldosterone-angiotensin system. Although ANP and BNP are generally considered to be cardioprotective, all these systems will need to be carefully monitored in patients receiving interventions that target natriuretic peptides. This monitoring should be complemented by ${ }^{18} \mathrm{~F}$-fluorodeoxyglucose PET imaging as well as careful indirect calorimetry. Indeed, studies involving comparisons of the effects of natriuretic peptide infusions in obese and lean individuals, as well as those with normal insulin sensitivity and insulin resistance, are already underway. ${ }^{72}$

If the results of such clinical studies support the notion that natriuretic peptides have therapeutic potential for promoting energy expenditure and weight loss, formulations of these peptides that retain adequate potency and have a long half-life should be developed, because native ANP and BNP have a half-life in the circulation of only $\sim 2 \mathrm{~min}$ and $20 \mathrm{~min}$, respectively. ${ }^{73-76}$ Some efforts have already been made to develop such agents for use in the cardiovascular setting. ${ }^{77,78}$ In individuals with obesity, the increased levels of NPR-C in adipose tissue might preclude the therapeutic use of natriuretic peptides to stimulate NPR-A. However, interventions that improve insulin sensitivity in these individuals might also reduce NPR-C levels and thereby enable natriuretic peptides to be efficacious. In addition, development of agents that can selectively block NPR-C or selectively activate NPR-A might be desirable in this setting.

\section{Conclusions}

The understanding of interorgan crosstalk is rapidly advancing as further examples of important physiological signalling systems are unveiled. However, with regard to understanding communication between the heart and adipose tissue, much work remains to be done. Studies to investigate the basic role of natriuretic peptides in metabolism-including defining the full range of their target tissues-are clearly needed. Furthermore, a detailed molecular understanding of natriuretic peptides needs to be obtained, including how the expression of their receptors is regulated at the transcriptional level, by phosphorylation and intracellular trafficking. Given the intimate association between metabolic and cardiovascular disease, investigators from these two fields will need to work closely together.

As noted by Thomas Wang, ${ }^{79}$ "Indeed, molecules that are capable of lowering blood pressure, preventing sodium retention, and reducing fat accumulation seem almost ideally suited for the fight against cardiometabolic disease."

\begin{tabular}{l} 
Review criteria \\
\hline PubMed and Novartis BioGPS (for gene expression) \\
databases were searched to identify articles for \\
inclusion in this Review. The search terms used included \\
"mitochondria", "brown adipose tissue", "UCP1", \\
"hypertension", "obesity", "gene transcription", "kinase", \\
"natriuretic", "adrenergic", and "uncoupling”. Internet \\
webinars of relevance to topics raised in this Review were \\
also audited. Papers chosen for inclusion were full-text \\
research articles from peer-reviewed, largely English- \\
language journals. The reference lists of key articles were \\
searched to identify further relevant articles. The patent \\
literature was also examined to search for possible \\
translational or therapeutic concepts or applications.
\end{tabular}

1. Caterson, I. D. et al. Maintained intentional weight loss reduces cardiovascular outcomes: results from the Sibutramine Cardiovascular OUTcomes (SCOUT) trial. Diabetes Obes. Metab. 14, 523-530 (2012).

2. Lepor, N. E., Fouchia, D. D. \& McCullough, P. A. New vistas for the treatment of obesity: turning the tide against the leading cause of morbidity and cardiovascular mortality in the developed world. Rev. Cardiovasc. Med. 14, 20-39; quiz 40 (2013).

3. Moro, C. \& Lafontan, M. Natriuretic peptides and cGMP signaling control of energy homeostasis. Am. J. Physiol. Heart Circ. Physiol. 304, H358-H368 (2013).
4. Sarzani, R., Dessi-Fulgheri, P., Paci, V. M., Espinosa, E. \& Rappelli, A. Expression of natriuretic peptide receptors in human adipose and other tissues. J. Endocrinol. Invest. 19, 581-585 (1996).

5. Dessi-Fulgheri, P. et al. Plasma atrial natriuretic peptide and natriuretic peptide receptor gene expression in adipose tissue of normotensive and hypertensive obese patients. J. Hypertens. 15, 1695-1699 (1997).

6. Wang, T. J. et al. Impact of obesity on plasma natriuretic peptide levels. Circulation 109, 594-600 (2004).

7. Dessi-Fulgheri, P., Sarzani, R. \& Rappelli, A. The natriuretic peptide system in obesity-related hypertension: new pathophysiological aspects. J. Nephrol. 11, 296-299 (1998).

8. Morton, N. M. et al. A stratified

transcriptomics analysis of polygenic fat and lean mouse adipose tissues identifies novel candidate obesity genes. PLOS ONE 6, e23944 (2011).

9. Sengenès, C., Berlan, M., De Glisezinski, I., Lafontan, M. \& Galitzky, J. Natriuretic peptides: a new lipolytic pathway in human adipocytes. FASEB J. 14, 1345-1351 (2000).

10. Birkenfeld, A. L. et al. Lipid mobilization with physiological atrial natriuretic peptide concentrations in humans. J. Clin. Endocrinol. Metab. 90, 3622-3628 (2005). 
11. Birkenfeld, A. L. et al. $\beta$-adrenergic and atrial natriuretic peptide interactions on human cardiovascular and metabolic regulation. J. Clin. Endocrinol. Metab. 91, 5069-5075 (2006).

12. Birkenfeld, A. L. et al. Atrial natriuretic peptide induces postprandial lipid oxidation in humans. Diabetes 57, 3199-3204 (2008).

13. Bordicchia, M. et al. Cardiac natriuretic peptides act via p38 MAPK to induce the brown fat thermogenic program in mouse and human adipocytes. J. Clin. Invest. 122, 1022-1036 (2012).

14. Engeli, S. et al. Natriuretic peptides enhance the oxidative capacity of human skeletal muscle. J. Clin. Invest. 122, 4675-4679 (2012).

15. Yehuda-Shnaidman, E., Buehrer, B., Pi, J., Kumar, N. \& Collins, S. Acute stimulation of white adipocyte respiration by PKA-induced lipolysis. Diabetes 59, 2474-2483 (2010).

16. Zurlo, F., Larson, K., Bogardus, C. \& Ravussin, E. Skeletal muscle metabolism is a major determinant of resting energy expenditure. J. Clin. Invest. 86, 1423-1427 (1990).

17. Bal, N. C. et al. Sarcolipin is a newly identified regulator of muscle-based thermogenesis in mammals. Nat. Med. 18, 1575-1579 (2012).

18. Sheldon, E. F. The so-called hibernating gland in mammals: A form of adipose tissue. Anat. Rec. 28, 331-347 (1924).

19. Aherne, W. \& Hull, D. The site of heat production in the newborn infant. Proc. R. Soc. Med. 57, 1172-1173 (1964).

20. Rothwell, N. J. \& Stock, M. J. A role for brown adipose tissue in diet-induced thermogenesis. Nature 281, 31-35 (1979).

21. Heaton, J. M. The distribution of brown adipose tissue in the human. J. Anat. 112, 35-39 (1972).

22. Lee, P., Swarbrick, M. M. \& Ho, K. K. Brown adipose tissue in adult humans: a metabolic renaissance. Endocr. Rev. 34, 413-438 (2013).

23. Nedergaard, J., Bengtsson, T. \& Cannon, B. Unexpected evidence for active brown adipose tissue in adult humans. Am. J. Physiol. Endocrinol. Metab. 293, E444-E452 (2007).

24. van Marken Lichtenbelt, W. D. et al. Coldactivated brown adipose tissue in healthy men. N. Engl. J. Med. 360, 1500-1508 (2009).

25. Cypess, A. M. et al. Identification and importance of brown adipose tissue in adult humans. N. Engl. J. Med. 360, 1509-1517 (2009).

26. Virtanen, K. A. et al. Functional brown adipose tissue in healthy adults. N. Engl. J. Med. 360 , 1518-1525 (2009).

27. Zingaretti, M. C. et al. The presence of UCP1 demonstrates that metabolically active adipose tissue in the neck of adult humans truly represents brown adipose tissue. FASEB J. 23 3113-3120 (2009).

28. Saito, M. et al. High incidence of metabolically active brown adipose tissue in healthy adult humans: effects of cold exposure and adiposity. Diabetes 58, 1526-1531 (2009).

29. Ouellet, V. et al. Brown adipose tissue oxidative metabolism contributes to energy expenditure during acute cold exposure in humans. J. Clin. Invest. 122, 545-552 (2012).

30. Orava, J. et al. Blunted metabolic responses to cold and insulin stimulation in brown adipose tissue of obese humans. Obesity (Silver Spring) http://dx.doi.org/10.1002/oby.20456.

31. Matthias, A. et al. Thermogenic responses in brown fat cells are fully UCP1-dependent. UCP2 or UCP3 do not substitute for UCP1 in adrenergically or fatty acid-induced thermogenesis. J. Biol. Chem. 275 , 25073-25081 (2000).
32. Cannon, B. \& Nedergaard, J. Brown adipose tissue: function and physiological significance. Physiol. Rev. 84, 277-359 (2004).

33. Wang, T., Zang, Y., Ling, W., Corkey, B. E. \& Guo, W. Metabolic partitioning of endogenous fatty acid in adipocytes. Obes. Res. 11, 880-887 (2003).

34. Maassen, J. A., Romijn, J. A. \& Heine, R. J. Fatty acid-induced mitochondrial uncoupling in adipocytes as a key protective factor against insulin resistance and beta cell dysfunction: do adipocytes consume sufficient amounts of oxygen to oxidise fatty acids? Diabetologia 51 , 907-908 (2008).

35. Stenson, B. M. et al. Activation of liver X receptor $(\mathrm{LXR})$ regulates substrate oxidation in white adipocytes. Endocrinology 150, 4103-4113 (2009).

36. Wilson-Fritch, L. et al. Mitochondrial remodeling in adipose tissue associated with obesity and treatment with rosiglitazone. J. Clin. Invest. 114, 1281-1289 (2004).

37. Kusminski, C. M. \& Scherer, P. E. Mitochondrial dysfunction in white adipose tissue. Trends Endocrinol. Metab. 23, 435-443 (2012).

38. Böttcher, H. \& Fürst, P. Decreased white fat cell thermogenesis in obese individuals. Int. J. Obes. Relat. Metab. Disord. 21, 439-444 (1997).

39. Landsberg, L. \& Young, J. B. The role of the sympathoadrenal system in modulating energy expenditure. J. Clin. Endocrinol. Metab. 13, 475-499 (1984).

40. Cousin, B. et al. Occurrence of brown adipocytes in rat white adipose tissue: molecular and morphological characterization. J. Cell Sci. 103, 931-942 (1992).

41. Wu, J. et al. Beige adipocytes are a distinct type of thermogenic fat cell in mouse and human. Cell 150, 366-376 (2012)

42. Cypess, A. M. et al. Anatomical localization, gene expression profiling and functional characterization of adult human neck brown fat. Nat. Med. 19, 635-639 (2013).

43. Jespersen, N. Z. et al. A classical brown adipose tissue mRNA signature partly overlaps with brite in the supraclavicular region of adult humans. Cell Metab. 17, 798-805 (2013).

44. Cao, W. et al. p38 mitogen-activated protein kinase is the central regulator of cyclic AMPdependent transcription of the brown fat uncoupling protein 1 gene. Mol. Cell. Biol. 24, 3057-3067 (2004).

45. Fregly, M. J., Kikta, D. C., Threatte, R. M., Torres, J. L. \& Barney, C. C. Development of hypertension in rats during chronic exposure to cold. J. Appl. Physiol. 66, 741-749 (1989).

46. Yuan, K. et al. Modification of atrial natriuretic peptide system in cold-induced hypertensive rats. Regul. Pept. 154, 112-120 (2009).

47. Sun, Z., Cade, J. R., Fregly, M. J. \& Rowland, N. E. Effect of chronic treatment with propranolol on the cardiovascular responses to chronic cold exposure. Physiol. Behav. 62, 379-384 (1997).

48. Granneman, J. G., Lahners, K. N. \& Chaudhry, A. Molecular cloning and expression of the rat $\beta_{3}$-adrenergic receptor. Mol. Pharmacol. 40, 895-899 (1991).

49. Nahmias, C. et al. Molecular characterization of the mouse $\beta_{3}$-adrenergic receptor: relationship with the atypical receptor of adipocytes. EMBO J. 10, 3721-3727 (1991).

50. Nakatsuji, H. et al. Reciprocal regulation of natriuretic peptide receptors by insulin in adipose cells. Biochem. Biophys. Res. Commun. 392, 100-105 (2010).

51. Pivovarova, O. et al. Insulin up-regulates natriuretic peptide clearance receptor expression in the subcutaneous fat depot in obese subjects: a missing link between CVD risk and obesity? J. Clin. Endocrinol. Metab. 97, E731-E739 (2012)

52. Potter, L. R. \& Hunter, T. Guanylyl cyclase-linked natriuretic peptide receptors: structure and regulation. J. Biol. Chem. 276, 6057-6060 (2001).

53. Kuhn, M. Structure, regulation, and function of mammalian membrane guanylyl cyclase receptors, with a focus on guanylyl cyclase-A. Circ. Res. 93, 700-709 (2003).

54. Sengenes, C. et al. Natriuretic peptidedependent lipolysis in fat cells is a primate specificity. Am. J. Physiol. Regul. Integr. Comp. Physiol. 283, R257-R265 (2002).

55. Shibasaki, I. et al. Greater expression of inflammatory cytokines, adrenomedullin, and natriuretic peptide receptor-C in epicardial adipose tissue in coronary artery disease. Regul. Pept. 165, 210-217 (2010).

56. Fitzgibbons, T. P. et al. Similarity of mouse perivascular and brown adipose tissues and their resistance to diet-induced inflammation. Am. J. Physiol. Heart Circ. Physiol. 301, H1425-H1437 (2011).

57. Kiefer, F. W., Cohen, P. \& Plutzky, J. Fifty shades of brown: perivascular fat, thermogenesis, and atherosclerosis. Circulation 126, 1012-1015 (2012).

58. Payne, G. A., Kohr, M. C. \& Tune, J. D. Epicardial perivascular adipose tissue as a therapeutic target in obesity-related coronary artery disease. Br. J. Pharmacol. 165, 659-669 (2012).

59. Chang, L. et al. Loss of perivascular adipose tissue on peroxisome proliferator-activated receptor- $\gamma$ deletion in smooth muscle cells impairs intravascular thermoregulation and enhances atherosclerosis. Circulation 126 , 1067-1078 (2012).

60. Anker, S. D. et al. Prognostic importance of weight loss in chronic heart failure and the effect of treatment with angiotensin-converting-enzyme inhibitors: an observational study. Lancet 361 1077-1083 (2003).

61. Anker, S. D. \& Sharma, R. The syndrome of cardiac cachexia. Int. J. Cardiol. 85, 51-66 (2002).

62. Pureza, V. \& Florea, V. G. Mechanisms for cachexia in heart failure. Curr. Heart Fail. Rep. http:// dx.doi.org/10.1007/s11897-013-0153-9.

63. Melenovsky, V. et al. Relationships between right ventricular function, body composition and prognosis in advanced heart failure. J. Am. Coll. Cardiol. 62, 1660-1670 (2013).

64. Christensen, H. M. et al. Prevalence of cachexia in chronic heart failure and characteristics of body composition and metabolic status. Endocrine 43, 626-634 (2013).

65. Szabó, T. et al. Increased catabolic activity in adipose tissue of patients with chronic heart failure. Eur. J. Heart Fail. 15, 1131-1137 (2013).

66. Polak, J. et al. Lipolytic effects of B-type natriuretic peptide 1-32 in adipose tissue of heart failure patients compared with healthy controls. J. Am. Coll. Cardiol. 58, 1119-1125 (2011).

67. Welsh, P. \& McMurray, J. J. B-type natriuretic peptide and glycaemia: an emerging cardiometabolic pathway? Diabetologia 55, 1240-1243 (2012).

68. Vijgen, G. H. et al. Increase in brown adipose tissue activity after weight loss in morbidly obese subjects. J. Clin. Endocrinol. Metab. 97, E1229-E1233 (2012).

69. Batal, O. et al. Effect of obesity on B-type natriuretic peptide levels in patients with pulmonary arterial hypertension. Am. J. Cardiol. 110, 909-914 (2012). 
70. Lippi, G., Schena, F. \& Guidi, G. C. Baseline and post-exercise $\mathrm{N}$-terminal pro-B-type natriuretic peptide values are associated with body mass index. Am. J. Cardiol. 109, 303-304 (2012).

71. Magnusson, M. et al. Low plasma level of atrial natriuretic peptide predicts development of diabetes: the prospective Malmo Diet and Cancer study. J. Clin. Endocrinol. Metab. 97, 638-645 (2012).

72. Translational Research Institute for Metabolism and Diabetes Research Studies: BNP [online] http://www.tri-md.org/research-studies/bnp (2013).

73. Luft, F. C. et al. Atriopeptin III kinetics and pharmacodynamics in normal and anephric rats. J. Pharmacol. Exp. Ther. 236, 416-418 (1986).
74. Nakao, K et al. The pharmacokinetics of a-human atrial natriuretic polypeptide in healthy subjects. Eur. J. Clin. Pharmacol. 31, 101-103 (1986).

75. Yandle, T. G. et al. Metabolic clearance rate and plasma half life of $\alpha$-human atrial natriuretic peptide in man. Life Sci. 38, 1827-1833 (1986).

76. Richards, A. M. et al. Brain natriuretic peptide: natriuretic and endocrine effects in essential hypertension. J. Hypertens. 11, 163-170 (1993).

77. Zakeri, R. \& Burnett, J. C. Designer natriuretic peptides: a vision for the future of heart failure therapeutics. Can. J. Physiol. Pharmacol. 89, 593-601 (2011).
78. de Bold, M. K. et al. Characterization of a longacting recombinant human serum albumin-atrial natriuretic factor (ANF) expressed in Pichia pastoris. Regul. Pept. 175, 7-10 (2012).

79. Wang, T. J. The natriuretic peptides and fat metabolism. N. Engl. J. Med. 367, 377-378 (2012).

80. Cypess, A. M. et al. Cold but not sympathomimetics activates human brown adipose tissue in vivo. Proc. Natl Acad. Sci. USA 109, 10001-10005 (2012).

81. Vosselman, M. J. et al. Systemic $\beta$-adrenergic stimulation of thermogenesis is not accompanied by brown adipose tissue activity in humans. Diabetes 61, 3106-3113 (2012). 